\title{
A PRÜFER TRANSFORMATION FOR NONSELFADJOINT SYSTEMS
}

\author{
KURT KREITH
}

\begin{abstract}
The Prüfer transformation has been generalized to selfadjoint matrix differential equations by $\mathrm{J}$. Barrett and others. Those results are extended to nonselfadjoint abstract systems of the form considered by Kamke in the scalar case and Reid in the matrix case.
\end{abstract}

Generalizations of the Prüfer transformation [1] to selfadjoint matrix differential systems of the form

$$
Y^{\prime}=B(x) Z ; \quad Z^{\prime}=-D(x) Y \quad(a \leqq x<\infty)
$$

have been studied by Barrett [2], Etgen [3], [4], and Reid [5], [6]. Hille [7] and Benson and the author [8] have established Prüfer transformations for abstract differential systems of the form (1) where $B(x), D(x), Y(x)$ and $Z(x)$ take their values in a Banach algebra $\mathcal{B}$. In all these studies the selfadjointness conditions $B(x) \equiv B^{*}(x) ; D(x) \equiv D^{*}(x)$ play an essential role. The purpose of this note is to establish a Prüfer transformation for nonselfadjoint $\mathfrak{B}$-valued matrix differential systems of the form

$$
Y^{\prime}=A(x) Y+B(x) Z, \quad Z^{\prime}=-D(x) Y+E(x) Z .
$$

Such a transformation has been established in the scalar case by Kamke [9], [10], and (by different techniques) in the matrix case by Reid [6].

It is assumed that $A(x), B(x), D(x)$, and $E(x)$ are strongly continuous $\beta$-valued functions on the semi-infinite interval $I=[a, \infty)$, and all derivatives below are to be taken in the strong topology. In passing from (1) to the more general system (2), we lose the relation $\left(Y^{*} Z-Z^{*} Y\right)^{\prime} \equiv 0$ which is used in constructing "prepared" or "conjoined" solutions in the selfadjoint case. As pointed out to the author by W. T. Reid, the condition that a solution of (1) be conjoined is analogous to restricting oneself to real solutions in the scalar case. Accordingly our Prüfer transformation for (2) is motivated by a study by Barrett [11] of a Prüfer transformation for complex valued solutions of a scalar system of the form (1).

Received by the editors January 4, 1971.

AMS 1969 subject classifications. Primary 3495; Secondary 3442.

Key words and phrases. Prüfer transformation, Banach algebra, nonselfadjoint systems, conjoined, $C^{*}$-algebra, Hilbert space. 
The abstract trigonometric functions $s(x ; a, Q), C(x ; a, Q), S(x ; a, Q)$, $c(x ; a, Q)$ will be defined as solutions of a system of the form

$$
\begin{aligned}
& s^{\prime}=Q(x) C ; \quad s(a)=0, \quad C^{\prime}=-Q^{*}(x) s ; \quad C(a)=E, \\
& S^{\prime}=Q^{*}(x) c ; \quad S(a)=0, \quad c^{\prime}=-Q(x) S ; \quad c(a)=E .
\end{aligned}
$$

Here $Q(x)$ is to be a strongly continuous $\mathfrak{B}$-valued function on $I$ and $E$ denotes the identity element in $\Re$. In case $Q(x)$ is nonsingular on $I$, $s(x ; a, Q)$ and $c(x ; a, Q)$ can be defined more directly as solutions of

and

$$
\left(Q^{-1}(x) s^{\prime}\right)^{\prime}+Q^{*}(x) s=0, \quad s(a)=0 ; \quad s^{\prime}(a)=Q(a),
$$

$$
\left(Q^{-1}(x) c^{\prime}\right)^{\prime}+Q^{*}(x) c=0, \quad c(a)=E ; \quad c^{\prime}(a)=0,
$$

respectively.

We shall make use of the trigonometric identities contained in the following lemmas.

LEMMA 1. If $s, c, S$, and $C$ satisfy (3) on I, then

(i) $s^{*} s+C^{*} C \equiv E$ on $I$;

(ii) $C^{*} S-s^{*} c \equiv 0$ on $I$.

Proof. A direct calculation using (3) shows that $\left(s^{*} s+C^{*} C\right)^{\prime} \equiv 0$ and $\left(C^{*} S-s^{*} c\right)^{\prime} \equiv 0$ on $I$. The result now follows readily from the initial conditions of (3).

LEMma 2. If $s, c, S$, and $C$ satisfy (3) on I, then

(i) $s s^{*}+c c^{*} \equiv E$ on $I$;

(ii) $S S^{*}+C C^{*} \equiv E$ on $I$;

(iii) $S c^{*}-C s^{*} \equiv 0$ on $I$;

(iv) $s C^{*}-c S^{*} \equiv 0$ on $I$.

Proof. Define $K=s s^{*}+c c^{*}, L=S S^{*}+C C^{*}, M=S c^{*}-C s^{*}$, and $N=s C^{*}-c S^{*}$. A direct calculation using (3) shows that $K, L, M$, and $N$ satisfy the system

$$
\begin{aligned}
K^{\prime}=N Q^{*}-Q M ; & K(a)=E, & L^{\prime}=M Q-Q^{*} N ; & L(a)=E \\
M^{\prime}=Q^{*} K-L Q^{*} ; & M(a)=0, & N^{\prime}=Q L-K Q ; & N(a)=0 .
\end{aligned}
$$

Since (4) has the obvious solution $K=L \equiv E, M=N \equiv 0$ and since solutions of (4) are unique, Lemma 2 is established.

Our Prüfer transformation will apply to a solution $Y(x), Z(x)$ of (2) satisfying $Y(a)=0, Z(a)$ nonsingular. We shall seek to represent such a solution in the form

$$
Y(x)=s^{*}(x ; a, Q) R(x) ; \quad Z(x)=c^{*}(x ; a, Q) R(x)
$$

by proper choice of $Q(x)$ and $R(x)$. 
If (5) is to be satisfied, then by (2)

$s^{*} R^{\prime}+C^{*} Q^{*} R=A s^{*} R+B c^{*} R, c^{*} R^{\prime}-S^{*} Q^{*} R=-D s^{*} R+E c^{*} R$.

Using Lemma 2 this system can be solved for $R^{\prime}$ and $Q^{*} R$ to yield

$$
\begin{gathered}
R^{\prime}=\left(s A s^{*}+s B c^{*}-c D a^{*}+c E c^{*}\right) R ; \quad R(a)=Z(a), \\
Q^{*} R=\left(C A s^{*}+C B c^{*}+S D s^{*}-S E c^{*}\right) R .
\end{gathered}
$$

Equation (7) suggests that $Q^{*}(x)$ should satisfy

$$
Q^{*}=C A s^{*}+C B c^{*}+S D s^{*}-S E c^{*}
$$

or

$$
Q=s A^{*} C^{*}+c B^{*} C^{*}+s D^{*} S^{*}-c E^{*} S^{*}
$$

Thus equations (6) and (7") are natural candidates for defining $Q(x)$ and $R(x)$ such that (5) is valid. A direct calculation shows that (6) and $\left(7^{\prime \prime}\right)$ do indeed validate (5). However, since $s, c, S$, and $C$ are all functions of $Q$, the solvability of $\left(7^{\prime \prime}\right)$ remains to be established. This problem can be circumvented as in [8] by using $\left(7^{\prime}\right)$ and $\left(7^{\prime \prime}\right)$ to define the trigonometric functions. Specifically, consider

$$
\begin{aligned}
s^{\prime} & =\left(s A^{*} C^{*}+c B^{*} C^{*}+s D^{*} S^{*}-C E^{*} S^{*}\right) C ; & s(a) & =0, \\
C^{\prime} & =-\left(C A s^{*}+C B c^{*}+S D s^{*}-S E c^{*}\right) s ; & C(a) & =E, \\
S^{\prime} & =\left(C A s^{*}+C B c^{*}+S D s^{*}-S E c^{*}\right) c ; & S(a) & =0, \\
c^{\prime} & =-\left(s A^{*} C^{*}+c B^{*} C^{*}+s D^{*} S^{*}-C E^{*} S^{*}\right) S ; & c(a) & =E .
\end{aligned}
$$

By standard existence theory the system $\left(3^{\prime}\right)$ has a unique solution $s(x)$, $C(x), S(x), c(x)$ near $x=a$, and this solution can be continued as long as it remains bounded in the norm for $\Re$ (see [8]). Our principal result is as follows.

THEOREM 1. Let $Y(x), Z(x)$ be a solution of (2) satisfying $Y(a)=0$, $Z(a)$ nonsingular. If $R(x)$, and $s(x), c(x)$ are solutions of $(6)$ and $\left(3^{\prime}\right)$, respectively, then $Y(x), Z(x)$ admits the Prüfer transformation

$$
Y(x)=s^{*}(x) R(x) ; \quad Z(x)=c^{*}(x) R(x) .
$$

Proof. It remains only to verify that the relations $\left(5^{\prime}\right)$ are compatible with (2). Using Lemma 1 we verify that

$$
\begin{aligned}
Y^{\prime}= & s^{*} R^{\prime}+s^{* \prime} R \\
= & s^{*}\left(s A s^{*}+s B c^{*}-c D s^{*}+c E c^{*}\right) R \\
& +C^{*}\left(C A s^{*}+C B c^{*}+S D s^{*}-S E c^{*}\right) R \\
= & \left(s^{*} s+C^{*} C\right)\left(A s^{*}+B c^{*}\right) R+\left(C^{*} S-s^{*} c\right)\left(D s^{*}-E c^{*}\right) R \\
= & A Y+B Z .
\end{aligned}
$$


An analogous calculation shows that $Z^{\prime}=-D Y+E Z$, and this completes the proof.

Theorem 1 establishes a local Prüfer transformation in the neighborhood of $x=a$. To show that this transformation is valid on $I=[a, \infty)$, it is only necessary to show that the solutions of $\left(3^{\prime}\right)$ remain bounded in $\mathcal{B}$ for $a \leqq x<\infty$. The following is a slight generalization of an analogous result for selfadjoint systems [8].

THEOREM 2. If $\mathfrak{B}$ is a $C^{*}$-algebra (i.e. an algebra of operators on a given Hilbert space $\mathfrak{H})$, then $|s(x)| \leqq 1,|C(x)| \leqq 1,|S(x)| \leqq 1$ and $|c(x)| \leqq 1$ for $a \leqq x<\infty$.

Proof. In the Hilbert space $\mathfrak{S}$ consider any solution $y(x), z(x)$ of the system

$$
y^{\prime}=Q(x) z ; \quad z^{\prime}=-Q^{*}(x) y \quad(a \leqq x<\infty)
$$

satisfying $y(a)=0,\|z(a)\|=1$. Then

$$
\begin{aligned}
\frac{d}{d x}\left(\|y\|^{2}+\|z\|^{2}\right) & =\left(y, y^{\prime}\right)+\left(y^{\prime}, y\right)+\left(z, z^{\prime}\right)+\left(z^{\prime}, z\right) \\
& =(y, Q z)+(Q z, y)-\left(z, Q^{*} y\right)-\left(Q^{*} y, z\right)=0
\end{aligned}
$$

so that

$$
\|y(x)\|^{2}+\|z(x)\|^{2} \equiv 1
$$

for $a \leqq x<\infty$. Now if $s(x), C(x), S(x), c(x)$ satisfy (3), then for any constant unit vector $e, y(x)=s(x) e, z(x)=C(x) e$ satisfy $(8)$ and

$$
\|s(x) e\|^{2}+\|C(x) e\|^{2} \equiv 1 .
$$

This shows that $|s(x)| \leqq 1$ and $|C(x)| \leqq 1$ on $I$. An analogous argument shows that $|S(x)| \leqq 1$ and $|c(x)| \leqq 1$ on $I$.

COROLlaRY. If (2) is a real or complex matrix system, then a solution $Y(x), Z(x)$ of (2) admits the Prüfer transformation $\left(5^{\prime}\right)$ on $I=[a, \infty)$.

\section{BIBLIOGRAPHY}

1. H. Prüfer, Neue Herleitung der Sturm-Liouvilleschen Reihenentwicklung stetiger Funktionen, Math. Ann. 95 (1926), 499-518.

2. J. H. Barrett, $A$ Prüfer transformation for matrix differential equations, Proc. Amer. Math. Soc. 8 (1957), 510-518. MR 19, 415.

3. G. J. Etgen, Oscillatory properties of certain nonlinear matrix differential systems of second order, Trans. Amer. Math. Soc. 122 (1966), 289-310. MR 32 \#7834.

4. - A note on trigonometric matrices, Proc. Amer. Math. Soc. 17 (1966), 1226-1232. MR 35 \#4504.

5. W. T. Reid, A Prüfer transformation for differential systems, Pacific J. Math. 8 (1958), 575-584. MR 20 \#5913. 
6. W. T. Reid, Generalized polar coordinate transformations for differential systems, Rocky Mt. J. Math. 1 (1971), 383-406.

7. E. Hille, Lectures on ordinary differential equations, Addison-Wesley, Reading, Mass., 1969. MR 40 \#2939.

8. D. C. Benson and K. Kreith, On abstract Prüfer transformations, Proc. Amer. Math. Soc. 26 (1970), 137-140.

9. E. Kamke, A new proof of Sturm's comparison theorems, Amer. Math. Monthly 46 (1939), 417-421. MR 1, 54.

10. — Über Sturm's Vergleichssätze für homogene lineare Differentialgleichungen zweiter Ordnung und Systeme von zwei Differentialgleichungen erster Ordnung, Math. Z. 47 (1942), 788-795. MR 7, 297.

11. J. H. Barrett, Second order complex differential equations with a real independent variable, Pacific J. Math. 8 (1958), 187-200. MR 20 \#4675.

Department of Mathematics, University of California, Davis, California 95616 